\title{
Delayed radiation myelopathy in patients with non- Hodgkin lymphoma: The importance of serial MR- imaging and PET-CT in differential diagnosis and surveillance
}

\author{
Chawar Hayoun ${ }^{1 *}$, María del Mar Jiménez de la Peña ${ }^{1}$, Ana Álvarez Vázquez ${ }^{1}$, Lidia Gómez Vicente ${ }^{2}$, Antonio Maldonado Suárez ${ }^{3}$ and \\ Vicente Martínez de Vega ${ }^{1}$ \\ ${ }^{1}$ Department of Diagnostic Imaging, Hospital Universitario Quirón Madrid. Madrid, Spain \\ ${ }^{2}$ Department of Neurology, Hospital Universitario Quirón Madrid. Madrid, Spain \\ ${ }^{3}$ Department of Nuclear Medicine, Hospital Universitario Quirón Madrid. Madrid, Spain
}

\begin{abstract}
Delayed radiation myelopathy (DRM) is a rare but serious complication grown at spinal cord after a certain period of application of radiotherapy (RT). Factors such the radiation dose and time between applications, affect to the incidence as well as the severity of myelopathy.

Serial Magnetic Resonance Images (MRI) showed spinal cord enlargement and the signal intensity were increased. MRI alterations can be maintained, changed or progressed over time. In case of progression, it must be discarded that the imaging findings are due to relapse of the patient's underlying disease. Positron Emission Tomography/Computed Tomography (PET/CT) studies take a very important place in differential diagnosis of both pathologies, myelopathy radiation changes and in relapse of the disease.

We reviewed the literature and we present two cases with patients diagnosed with B-cell low-grade non-Hodgkin lymphoma and who received chemotherapy and radiotherapy below the limit agreed in the guidelines. Both patients developed progressive lower extremity weakness and MRI abnormalities of the spinal cord limited to the radiation field appeared.
\end{abstract}

\section{Cases}

Radiation therapy is one of the most important mainstay treatment modalities for a non-Hodgkin lymphoma with bone involvement.

In the last years, we observed two female patients who developed a severe neurological disorder due to the irradiation of vertebral bodies infiltrated by B-cell low-grade non-Hodgkin lymphoma.

The first patient, a 64-years-old woman, diagnosed with lymphoma with supra and infradiaphragmatic lymphadenopathies, and epidural masses in T12-L1 and L2-L3 (Figure 1).

She received intratecal chemotherapy for central nervous system (CNS) prophilaxis, followed by six cycles of CHOP-R chemotherapy (cyclophosphamide, doxorubicin, vincristine, prednisone and rituximab) and radiotherapy on epidural masses, with fields covering from T10 to L1. The calculated dose delivered to the spinal cord was 30 Gy in 2 Gy equivalent fractions and was completed in 15 days without unplanned breaks. The patient tolerated the treatment well, with limited mucositis and gastroenteritis. Complete remission was shown in the subsequent PET-CT and MRI controls.

The patient stayed well for approximately 20 months, when she began to show numbness and weakness of both lower limbs, with progressively worsening. Neurological examination revealed hyperreflexia and clonnus on lower limbs, sensitive deficit below the infraumbilical region, and gait disorder with sensitive ataxia and increased support base. On the following months, she experienced progressive worsening of ambulatory deficit, due to leg paresis.

Magnetic resonance imaging of the spinal cord demonstrated intramedullary lesion extending from T11 to the upper end of L1. The spinal cord appeared diffusely enlarged over the involved segment and a ring enhancing lesion was observed after Gadolinium administration (Figure 2). Suspected diagnoses were relapse lymphoma, primary intramedullary tumor, or radiation myelitis. The spinal tap revealed a normal CSF chemistry and microscopy.

For PET/CT examination, the patient was intravenously injected of F-18 fluorodeoxyglucose (F-18 FDG). There were no pathological FDG uptake suggesting malignancy at the spinal cord in the thoracic or lumbar region. According to patient history, clinical and radiological findings, the patient was diagnosed as DRM.

The patient was treated with high dose methylprednisolone, 500 $\mathrm{mg} /$ day for three days followed by oral methylprednisolone for one month. Rehabilitation and hyperbaric oxygen therapy were applied too.

*Correspondence to: Chawar Hayoun, Department of Diagnostic Imaging, Hospital Universitario Quirón Madrid, Madrid, Spain, E-mail: chawarhayoun@gmail.com

Received: May 05, 2018; Accepted: May 29, 2018; Published: June 01, 2018 


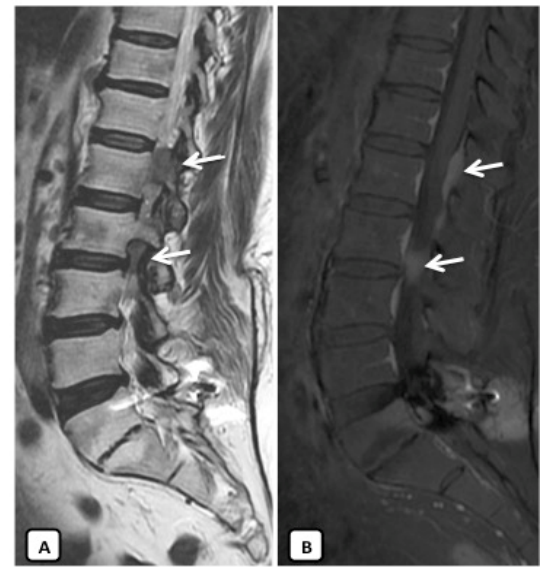

Figure 1. Saggital T2WI (A) and saggital $\mathrm{T} 1 \mathrm{WI}+\mathrm{Gd}+$ fat saturation (B). A fusiform isointense lesion located in the posterior epidural space in T12-L1 and L2-L3 on T2WI MRI (arrow). Homogeneous enhancement of the lesion was found after gadolinium administration (arrow)
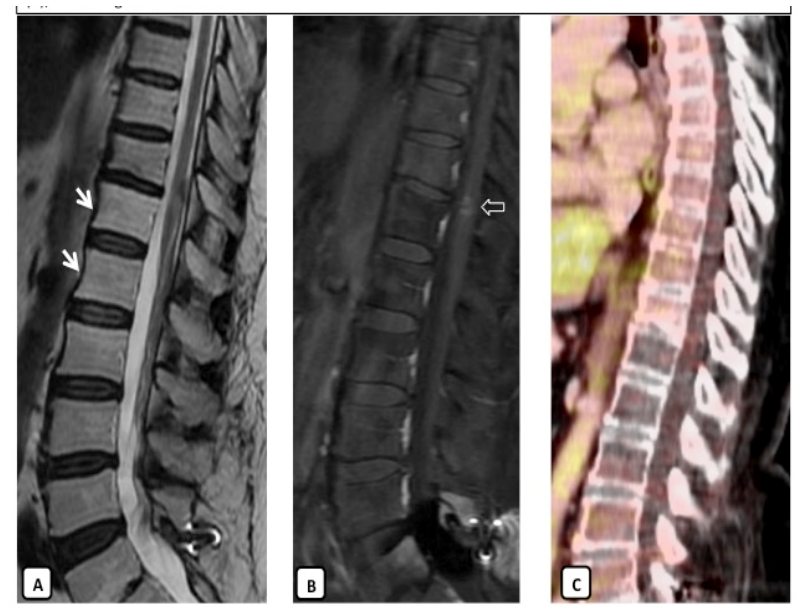

Figure 2. Findings after treatment with radiotherapy. Absence of epidural mass on T2WI (A) and T1WI $+\mathrm{Gd}$, with changes of fatty replacement of the vertebral bodies on saggital T2WI. Saggital T1 post gadolinium MRI (B) showing the area of enhancement within the cord (open arrow) and on the T2-WI (A) showing edema in the cord above and below the lesion (arrows). Absence of FDG uptake in the spinal cord on PET-CT images (C) indicating radio necrosis

She presents a transient response with slight clinical improvement, mainly of the sensorial affectation. Subsequent serial MRI studies confirmed radiological improvement and stable heterogenous enhancement in the spinal cord, without new areas of involvement (Figure 3). However, 4 months later, the patient was paraplegic, lost bladder and bowel function, and shown a mild increase in the craniocaudal extension of the enhanced lesion in the MRI images (Figure 4).

The second patient, aged 81 years, was diagnosed of lymphoma by vertebral mass biopsy in L1. In the initial MRI diagnosis, was evidenced a mass in L1 which breaks the posterior cortex of the vertebral body, extending to an anterior epidural mass that compromises the spinal canal, and other smaller lesions in D8 and D9, limited to the vertebral bodies (Figure 5). According the age of the patient, she underwent initial treatment with RT on the mass, followed by systemic chemotherapy. Subsequently, she continued treatment with Rituximab. 24 months after $\mathrm{RT}$, the patient shown a progressive decline in neurological function, dysesthesia and motor deficit in both lower limbs and sensory deficit below the T7 level. Autonomous ambulation was not possible.
Spinal cord MRI revealed a long segment of intramedullary lesion extended from T6 to T11 levels. The spinal cord appeared diffusely enlarged over the involved segment and a partially enhancing lesion was observed after Gadolinium administration. It was also evidenced, another focus of right paramedian nodular enhancement in the medullary cone. Supplementary PET is performed on suspicion of tumor involvement of the spinal cord, which is negative (Figure 6). A new MRI 3 months later, was revealed an edema reduction and stability of the enhancing lesion. (Figure 7).

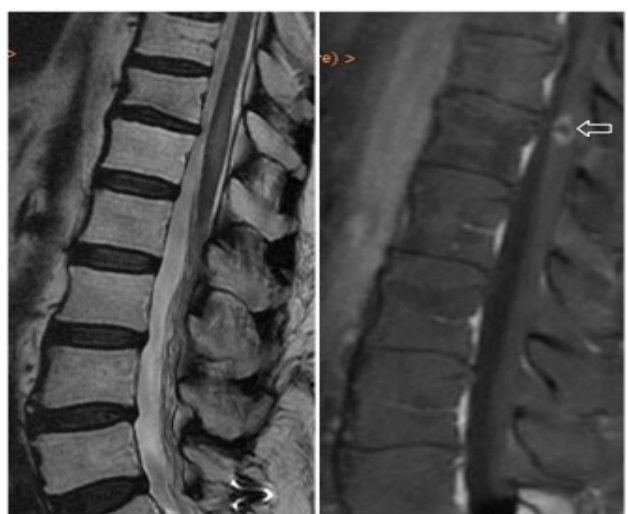

Figure 3. Six months after starting steroid therapy, she had gradual clinical improvement and the MRI shows no significant changes in signal intensity with a stability of the nodular enhancement in the spinal cord (open arrow)

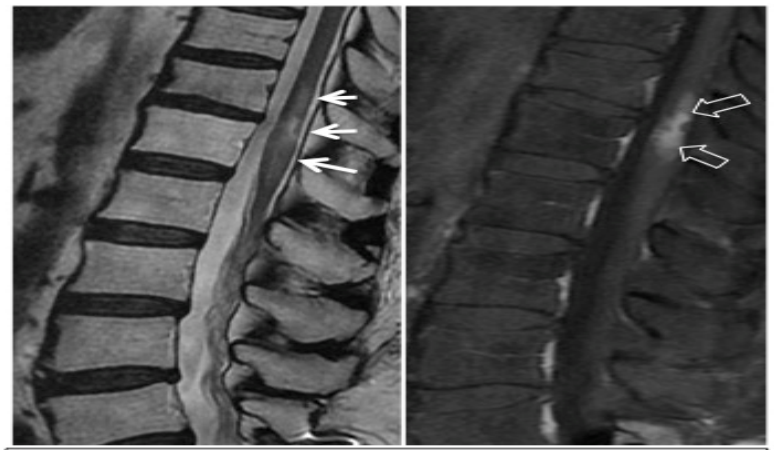

Figure 4. 4 months later, the patient presented clinical worsening, a new MRI demonstrated radiological progression of radionecrosis, with increased in density and craniocaudal extension of the enhancement (open arrows), and greater associated edema (arrows)
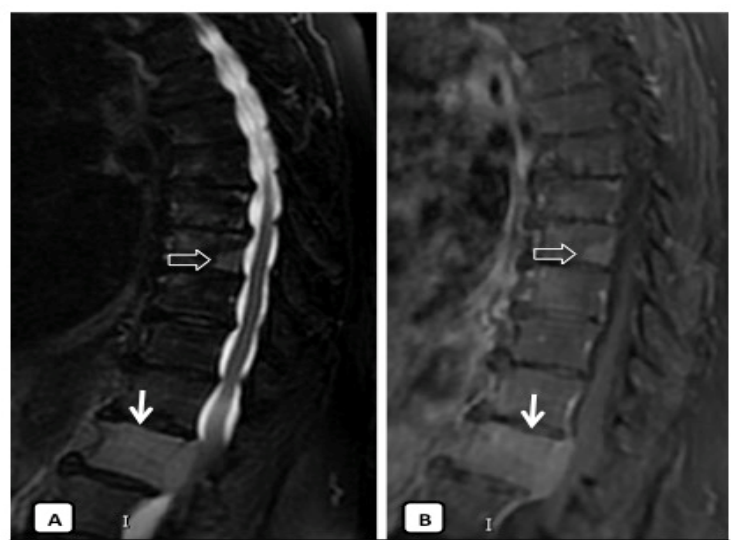

Figure 5. Sagittal T2WI (A) and contrast-enhanced sagittal T1WI (B): Expansive lesion in L1 vertebral body, which breaks the posterior cortex by associating an anterior epidural mass and compresses the medullary cord (arrow). Lesions with similar characteristics are also identified in vertebral bodies $\mathrm{T} 8$ and $\mathrm{T} 9$ (open arrow) 


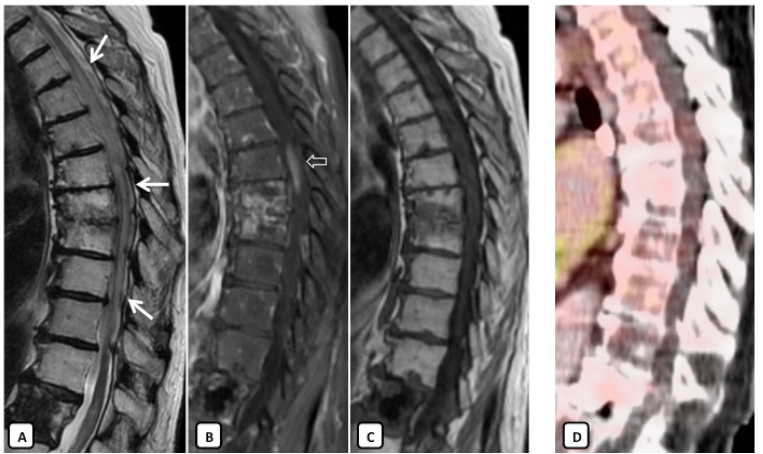

Figure 6. MRI performed 24 months after radiotherapy. MRI showed abnormal high signal intensity (arrows) from affecting a large segment of spinal cord from D4-D5 to D11-D12 on T2WI (A) and focal ovoid zone of contrast enhancement within the central cord at T7T8 (open arrow on B). Post-radiotherapy fatty marrow change can be seen in the adjacen vertebral bodies with subchondral sclerosis and enhancement on T1WI (C) and T1 post gadolinium (B). No uptake is seen in the PET-CT image (D) so the findings indicate radio necrosis

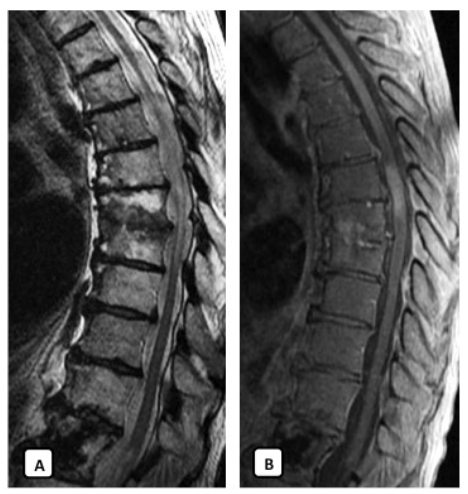

Figure 7. Three months later, MRI showed a significant reduction in the craniocaudal extension of the medullary edema on T2WI limited to D6 -D10. Sagittal T1WI after gadolinium confirmed stable enhancement in the spinal cord

\section{Discussion}

Neurologic complications after RT and/or chemotherapy for systemic cancer are not rare. The spinal cord is considered one of the most critical dose-limiting organs, also referred to as organs-at-risk $[1,2]$.

Total radiation dose, dose per fraction, time between RT applications, length of the spinal cord irradiation and the association with other systemic therapy affect the probability of the onset and severity of myelopathy. It is widely accepted that the risk of permanent spinal cord injury is very low $(0.03-0.2 \%)$, if it does not exceed a total of 45-50 Gy in 1.8-2 Gy daily fractions [3-5].

Exposure of the spinal cord to therapeutic radiation carries the risk of injury or damage [6], and are classified into three categories according to the clinical spectrum:

1. Early injury: After very high doses to the brain such as in nuclear accidents. Any acute clinical deterioration is generally related to increased tumor as in the context of extradural cord compression. There is no clinical equivalent of acute CNS syndrome and after a large single dose to the cord.

2. Subacute (self-limiting) myelopathy: it is the most frequent form of toxicity of the spinal cord by radiotherapy. It occurs after a latent period of 2 to 4 months and is characterized by parestesia in the back extremities and resolves spontaneously in a few months. It is considered to be produced by a transient demyelination of the medullary posterior cords, so it does not require treatment. Its development does not predict the appearance of chronic myelopathy in the future [6].

3. Late injury Delayed radiation myelopathy (DMR): Chronic progressive radiation myelopathy is typically irreversible and it's the purpose of our article [7,8].

DRM is a rare but feared complication of therapeutic radiation exposure to the spine cord, due to its progressive course and irreversibility [9]. Criteria used generally include the following: radiation therapy to the spinal cord, neurologic symptoms must correspond to the involved segment of spinal cord irradiated $[1,3,10]$, lack of neoplastic disease involving the cord in patients with normal cerebro-spinal fluid (CSF), and there must be a latency period of more than six months [11].

The neurological examination shows: sensory loss below the level of RT, slowly progressing ascending paresis and paralysis, mild hyperreflexia, bowel and bladder sphincter disturbances. Since antemortem confirmation of DRM is impossible, it often remains a diagnosis of exclusion $[12,14]$.

The MRI is currently the most widely used imaging tool in the diagnostic evaluation of radiation myelopathy [13]. As it is a rare complication, we only find in the literature, reports of isolated cases, with punctual images. In our study, we presented two cases with the same pathology and their follow up over time, with MRI, to see the evolution of the findings.

Characteristic MRI changes [14-16] include areas of low signals on T1-weighted images, swelling and diffuse high signal on T2 and enhancement in post-gadolinium $\mathrm{T} 1$-weighted images. The enhancement may be nodular, patched or ring-shaped, and confined to the area exposed to radiation. The fatty marrow change in the adjacent vertebral bodies may be the only clue to diagnosis [11], if the antecedent of previous radiotherapy treatment is unknown. As in the case of cerebral radio necrosis, MRI findings may vary along the time [17].

The central cord swelling seen on T2 weighted images may well represent an edema, produced in response to altered vascular permeability after radiation damage to the vascular endothelium $[15,18]$. Consequently, the edema can be significantly reduced with corticosteroid treatment.

Peripheral ring enhancement of the cord after gadolinium administration shows the localization of the major focus of cord damage, the point where is broken the blood-brain barrier $[8,18,19]$. This alteration can vary and increase in size, becoming more heterogeneous with imprecise borders with the appearance of "Swiss-cheese-like". This increase in size, is a result of diffuse white matter demyelination and necrosis and it must not be confused with medullary infiltration by the patient's disease [20], being very useful, the realization of PET-CT.

The PET-CT with fluorodeoxyglucose (FDG), plays an important role in the differential diagnosis from primary spinal cord lesions and spinal metastases [16,21]. Most malignant tumors show increased uptake of FDG, because tumor cell growth and malignant transformation are associated with increased hexokinase activity and over expression of glucose transporters. Therefore, in these entities, the PET-CT show a clearly decreased FDG uptake.

The systemic cytotoxic therapy received by our patients, in both cases they received Rituximab, in combination with radiotherapy, 
Hayoun C (2018) Delayed radiation myelopathy in patients with non-Hodgkin lymphoma: The importance of serial MR-imaging and PET-CT in differential diagnosis and surveillance

may be a factor that potentiates the observed radiation neurotoxicity. Although DRM has been observed following intrathecal chemotherapy with craniospinal irradiation in children, but there are very few cases and no clear association has been established [11]. Research in this field should continue, with more representative samples.

No treatment was shown to affect the progressive course of neurological situation in the treatment of DRM, the damage is irreversible, and the treatment is mostly supportive.

Some patients have acquired a short-term benefit from steroids, which may be related to the edema and inflammation. There have been reports in the literature, cases of patients who benefited from warfarin, pentoxifylline, vitamin $\mathrm{E}$, and hyperbaric oxygen treatments. In view of the low risk of side effects of steroids, hyperbaric oxygen and rehabilitation, a combination of these three treatments may be proposed [16,22].

\section{Conclusion}

Radiation induced complications, are usually delayed and may occur from months to years after irradiation. Delayed radiation myelopathy is an infrequent but very serious complication, because it has an irreversible progressive course and there is no effective treatment. Suspicion criteria are based on patient's neurological symptoms, history of radiotherapy on the spinal cord, and imaging findings. MRI is the best imaging tool in the diagnostic assessment and monitoring of radiation myelopathy, and the PET-CT can help in case of the diagnosis is not clear.

\section{References}

1. Wara WM, Phillips TL, Sheline GE, Schwade JG (1975) Radiation tolerance of the spinal cord. Cancer 35: 1558-1562. [Crossref]

2. Kim YH, Fayos JV (1981) Radiation tolerance of the cervical spinal cord. Radiology 139: 473-478. [Crossref]

3. Schultheiss TE (2008) The radiation dose-response of the human spinal cord. Int $J$ Radiat Oncol Biol Phys 71: 1455-1459. [Crossref]

4. Marcus RB Jr, Million RR (1990) The incidence of myelitis after irradiation of the cervical spinal cord. Int J Radiat Oncol Biol Phys 19: 3-8. [Crossref]

5. Van der Kogel AJ (1993) Retreatment tolerance of the spinal cord. Int J Radiat Oncol Biol Phys 26: 715-717. [Crossref]
6. Dropcho EJ (2010) Neurotoxicity of radiation therapy. Neurol Clin 28: 217-234. [Crossref]

7. Mul VE, de Jong JM, Murrer LH, van den Ende PL, Houben RM, et al. (2012) Lhermitte sign and myelopathy after irradiation of the cervical spinal cord in radiotherapy treatment of head and neck cancer. Strahlenther Onkol 188: 71-76. [Crossref]

8. Wong CS, Van Dyk J, Milosevic M, Laperriere NJ (1994) Radiation myelopathy following single courses of radiotherapy and retreatment. Int J Radiat Oncol Biol Phys 30: 575-581. [Crossref]

9. Gibbs IC, Patil C, Gerszten PC, Adler JR, Burton SA (2009) Delayed radiation-induced myelopathy after spinal radiosurgery. Neurosurgery 64: A67-72. [Crossref]

10. Palmer JJ (1972) Radiation myelopathy. Brain 95:109-122.

11. Schwartz DL, Schechter GP, Seltzer S, Chauncey TR (2000) Radiation myelitis following allogenic stem cell transplantation and consolidation radiotherapy for nonHodgkin's lymphoma. Bone Marrow Transplantation 26: 1355-1359. [Crossref]

12. Choucair AK (1991) Myelopathies in the cancer patient: incidence, presentation, diagnosis and management. Oncology 191 5: 25-31. [Crossref]

13. Wang PY, Shen WC (1991) Magnetic resonance imaging in two patients with radiation myelopathy. J Formosan Med Assoc 90: 583-585. [Crossref]

14. Wang PY, Shen WC, Jan JS (1995) Serial MRI changes in radiation myelopathy. Neuroradiology 37: 374-377. [Crossref]

15. Wong CS, Fehlings MG, Sahgal A (2015) Pathobiology of radiation myelopathy and strategies to mitigate injury. Spinal Cord 53: 574-580. [Crossref]

16. Pompili A, Crispo F, Raus L, Telera S, Vidiri A (2011) Symptomatic spinal cord necrosis after irradiation for vertebral metastatic breast cancer. J Clin Oncol 29: e53-e56. [Crossref]

17. Wang PY, Shen WC, Jan JS (1995) Serial MRI changes in radiation myelopathy Neuroradiology 37: 374-377. [Crossref]

18. Philippens ME, Gambarota G, van der Kogel AJ, Heerschap A (2009) Radiation effects in the rat spinal cord: evaluation with apparent diffusion coefficient versus $\mathrm{T} 2$ at serial MR imaging. Radiology 250: 387-397. [Crossref]

19. Michikawa M, Wada Y, Sano M, Uchihara T, Furukawa T, et al. (1991) Radiation myelopathy: significance of gadolinium-DTPA enhancement in diagnosis. Neuroradiology 33: 286-289. [Crossref]

20. Yasui T, Yagura H, Komiyama M, Fu Y, Nagata Y, et al. (1992) Significance of gadolinium-enhanced magnetic resonance imaging in differentiating spinal cord radiation myelopathy from tumor. $J$ Neurosurg 77: 628-631. [Crossref]

21. Uchida K, Nakajima H, Takamura T, Kobayashi S, Tsuchida T, et al. (2009) Neurological improvement associated with resolution of irradiation-induced myelopathy: serial magnetic resonance imaging and positron emission tomography findings. J Neuroimag 19: 274-276. [Crossref]

22. Calabró F, Jinkins JR (2000) MRI of radiation myelitis: A report of a case treated with hyperbaric oxygen. Eur Radiol 10: 1079-1084. [Crossref]

Copyright: $\odot 2018$ Hayoun C. This is an open-access article distributed under the terms of the Creative Commons Attribution License, which permits unrestricted use, distribution, and reproduction in any medium, provided the original author and source are credited. 